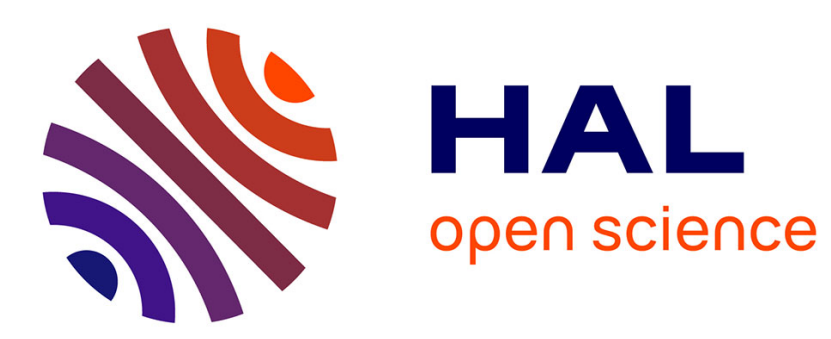

\title{
Influence de la position de l'outil sur le comportement dynamique en fraisage de parois minces
}

Vincent Thévenot, Lionel Arnaud, Gilles Dessein, Gilles Cazenave-Larroche

\section{To cite this version:}

Vincent Thévenot, Lionel Arnaud, Gilles Dessein, Gilles Cazenave-Larroche. Influence de la position de l'outil sur le comportement dynamique en fraisage de parois minces. Mechanics \& Industry, 2005, vol. 6, pp. 403-410. 10.1051/meca:2005048. hal-00800368

\section{HAL Id: hal-00800368 https://hal.science/hal-00800368}

Submitted on 13 Mar 2013

HAL is a multi-disciplinary open access archive for the deposit and dissemination of scientific research documents, whether they are published or not. The documents may come from teaching and research institutions in France or abroad, or from public or private research centers.
L'archive ouverte pluridisciplinaire HAL, est destinée au dépôt et à la diffusion de documents scientifiques de niveau recherche, publiés ou non, émanant des établissements d'enseignement et de recherche français ou étrangers, des laboratoires publics ou privés. 


\title{
Influence de la position de l'outil sur le comportement dynamique en fraisage de parois minces
}

\author{
Vincent Thevenot $^{1, a}$, Lionel Arnaud ${ }^{2}$, Gilles Dessein $^{2}$ et Gilles Cazenave-Larroche ${ }^{3}$ \\ 1 Turbomeca, ENIT 47, avenue d'Azereix, BP 1629, 65000 Tarbes Cedex, France \\ 2 Laboratoire Génie de Production, École Nationale d'Ingénieurs de Tarbes, ENIT, 47 avenue d'Azereix, BP 1629, \\ 65000 Tarbes Cedex, France \\ 3 Turbomeca, avenue Szydlowski, 64511 Bordes Cedex, France
}

\begin{abstract}
Résumé - Les problèmes vibratoires rencontrés lors de l'usinage en contournage de parois minces affectent la qualité de la pièce finie et dans une moindre mesure, la durée de vie de l'outil et de la broche. C'est pourquoi il est nécessaire de pouvoir limiter ces problèmes par un choix approprié des conditions de coupe. La théorie des lobes de stabilité permet de choisir les conditions de coupe adéquates en fonction du comportement dynamique de la pièce ou de l'outil. Nous introduisons ici les variations de comportement dynamique dues à la position de l'outil, dans le but d'obtenir des conditions de coupe optimales tout le long de l'usinage. Cette généralisation du tracé classique des lobes de stabilité nous conduit à représenter les lobes en trois dimensions. Ces résultats théoriques sont comparés à des usinages expérimentaux de pièces minces, en avalant.
\end{abstract}

Mots clés : Lobes de stabilité 3D / fraisage de paroi mince / comportement dynamique

\begin{abstract}
Influence of the tool position on the dynamic behaviour in milling of thin walled structure. Vibratory problems encountered when milling a thin walled structure affect the surface quality of the finished workpiece and to a lesser extent, the life of the tool and the spindle. This is why, it is necessary to be able to limit these problems by an appropriate choice of the cutting conditions. The stability lobes theory allows to choose the suitable cutting conditions according to the dynamic behaviour of the workpiece and the tool. We introduce here the variations of dynamic behaviour due to the tool position, in order to obtain optimum cutting conditions all along the machining. This general implementation of the classical stability lobes graph leads us to represent the lobes in three dimensions. These theoretical results are compared with experimental milling of thin walled structure, in down-milling.
\end{abstract}

Key words: 3D stability lobes / milling of thin walled structure / dynamic behaviour

\section{Introduction}

Lors de certaines opérations d'usinage, il peut apparaître des vibrations entre la pièce et l'outil. C'est notamment le cas en fraisage lorsqu'on utilise des outils longs de faible diamètre ou que l'on veut usiner une paroi fine en contournage. Ce phénomène engendre une imprécision dimensionnelle, géométrique et un mauvais état de surface (Fig. 1). On observe également une diminution de la durée de vie de l'outil et de la broche. C'est pourquoi il faut être capable de comprendre le phénomène, et de le maîtriser. Les bases de la compréhension des vibrations du couple outil-pièce

\footnotetext{
a Auteur pour correspondance :

vincent.thevenot@enit.fr
}

remontent aux années 1950 avec S.A. Tobias [1], J. Tlusty [2] et H.E. Merrit [3], qui ont expliqué le phénomène de vibrations auto-entretenues en coupe orthogonale, et mis au point la théorie des lobes de stabilité. Cette théorie permet, pour une vitesse de rotation donnée, de prédire la longueur d'arête en prise à partir de laquelle les vibrations vont apparaître. Ce premier modèle est particulièrement bien adapté aux vibrations de l'outil en tournage où la direction des efforts de coupe, l'épaisseur de copeau et les paramètres dynamiques ne changent pas notablement au cours du temps. La théorie des lobes de stabilité appliquée au fraisage orthogonal (outil sans angle d'hélice) apparaît à la fin des années $60[4,5]$, et est utilisée pour étudier les vibrations de l'outil. Au milieu des années 90, Y. Altintas [6] présente une forme analytique de la théorie des lobes de 


\section{Nomenclature}

\begin{tabular}{|ll|}
\hline$\alpha_{y y}$ & coefficient directionnel dynamique suivant $y$ \\
$\alpha_{i}(t)$ & fonction poids du mode considéré \\
$\phi_{\mathrm{ex}}$ & angle de sortie \\
$\phi_{\mathrm{st}}$ & angle d'entrée \\
$\underline{\Phi}_{i}(\underline{x})$ & forme propre du mode considéré \\
$\xi$ & taux d'amortissement \\
$\xi_{i}$ & taux d'amortissement du mode considéré \\
$\omega_{\mathrm{c}}$ & pulsation de broutement \\
$\omega_{0}$ & pulsation propre \\
$A_{\mathrm{e}}$ & profondeur de passe radiale \\
$A_{\mathrm{p}}$ & profondeur de passe axiale \\
$\underline{F}_{\mathrm{c}}$ & effort de coupe \\
$F_{\mathrm{r}}$ & effort radial \\
$F_{\mathrm{t}}$ & effort tangentiel \\
$G_{y}\left(i \omega_{\mathrm{c}}\right)$ & fonction de transfert du système usinant suivant $y$ \\
$k$ & raideur \\
$k_{\mathrm{i}}$ & raideur modale du mode considéré \\
$K_{\mathrm{r}}$ & coefficient spécifique de coupe radial \\
$K_{\mathrm{t}}$ & coefficient spécifique de coupe tangentiel \\
$m$ & nombre de longueurs d'onde entières entre deux passages de dent \\
$m_{\mathrm{i}}$ & masse modale du mode considéré \\
$M$ & point d'application de l'effort de coupe \\
$M_{0}$ & point de normalisation de la déformée propre du mode considéré \\
$n$ & nombre de modes en considération \\
$N$ & vitesse de rotation de la broche \\
$\underline{u}(\underline{x}, t)$ & champ de déplacement de la pièce considérée déformable \\
$U(t)$ & déplacement de la pièce assimilée à un corps rigide \\
$z$ & nombre de dents de l'outil \\
\hline
\end{tabular}

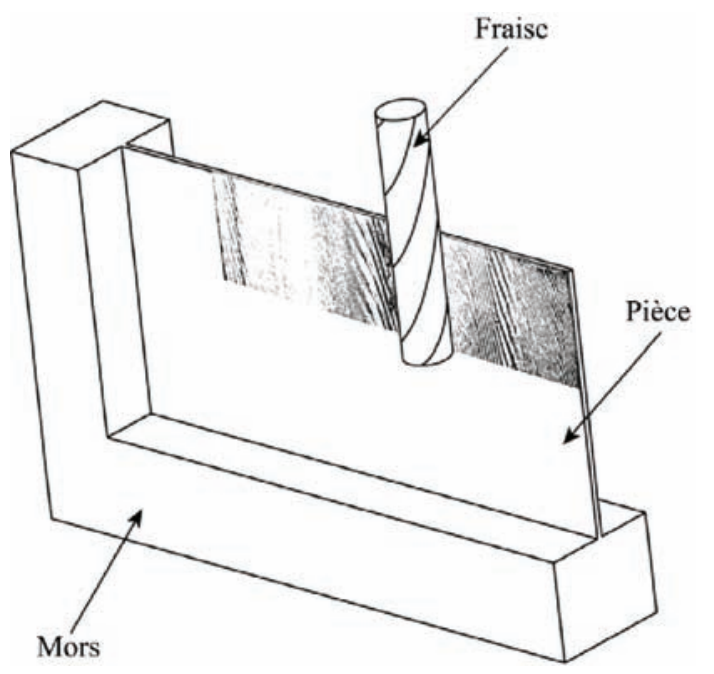

Fig. 1. Problèmes vibratoires rencontrés en contournage. compte que le comportement dynamique du couple outilpièce varie suivant la position de l'outil sur la pièce $[7,8]$. Nous montrerons dans cet article comment intégrer les variations de comportement de la pièce pendant l'usinage.

\section{Théorie des lobes de stabilité}

Dans ce travail, nous nous baserons essentiellement sur les travaux de Y. Altintas et E. Budak [6,9,10]. Nous ne donnons ici que les équations permettant de tracer les lobes.

La théorie des lobes de stabilité est basée sur un modèle de coupe avec vibrations régénératives. Elle vise à prédire dans quelles conditions (profondeur de passe axiale et vitesse de rotation broche) les oscillations dues à la dent $n+1$ ont une amplitude plus ou moins importante par rapport aux oscillations dues à la dent $n$ (Fig. 2).

\subsection{Hypothèses de départ}

- Nous considérons ici la pièce mobile par rapport à l'outil, qui est beaucoup plus rigide que la pièce. pour l'étude des vibrations de l'outil car ses caractéristiques dynamiques ne changent pas au cours du temps. Mais lorsqu'on usine une paroi fine, on se rend 


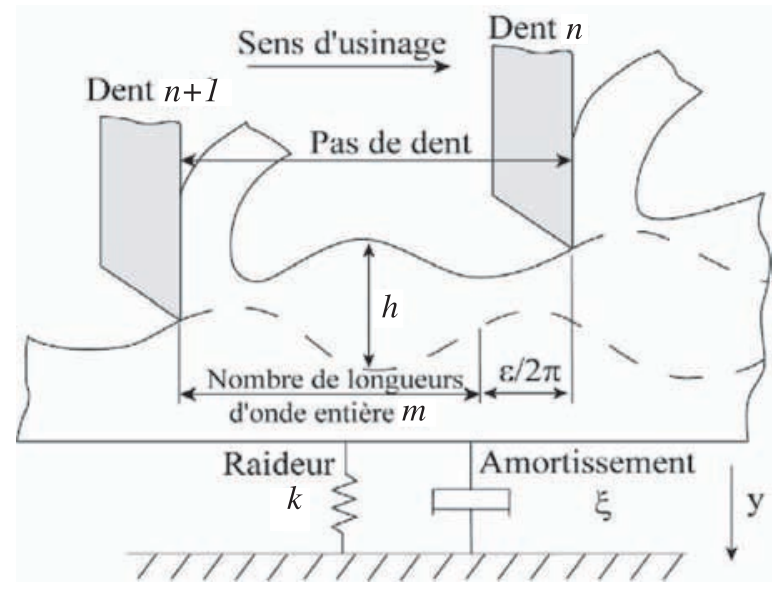

Fig. 2. Modèle de coupe avec vibrations régénératives à un degré de liberté.

- La pièce est assimilée à un corps rigide dans la zone d'usinage.

- L'enlèvement de matière est négligé.

- La pièce se déplace suivant la direction $y$, comme un corps rigide dans la zone usinée, et selon l'équation suivante :

$$
\ddot{U}+2 \xi \omega_{0} \dot{U}+\omega_{0}^{2} U=\left(\left(\underline{F}_{c} \cdot \underline{y}\right) \omega_{0}^{2}\right) / k
$$

- Nous utilisons une loi de coupe linéaire du type $F_{\mathrm{t}}=K_{\mathrm{t}} A_{\mathrm{p}} A_{\mathrm{e}}$ et $F_{\mathrm{r}}=K_{\mathrm{r}} F_{\mathrm{t}}$, où $F_{\mathrm{t}}$ et $F_{\mathrm{r}}$ sont respectivement les efforts de coupe tangentiels et radiaux, $K_{t}$ et $K_{\mathrm{r}}$ les coefficients spécifiques de coupe tangentiels et radiaux et $A_{\mathrm{p}}$ et $A_{\mathrm{e}}$ les profondeurs de passe axiales et radiales. Nous éviterons les cas où $A_{\mathrm{e}} \ll f_{\mathrm{z}}$, l'avance à la dent, pour lesquels la loi de coupe linéaire est peu précise [7]. C'est l'hypothèse la plus difficile à respecter étant donné l'imprécision sur la profondeur de passe radiale réelle en finition qui est très faible.

Ces hypothèses sont simples, notre but étant de montrer comment construire les lobes. Toutefois, cette construction est applicable à des modèles plus compliqués (loi de coupe non-linéaire, plusieurs degrés de liberté, simulation temporelle ...).

\subsection{Calcul de la profondeur de passe axiale limite en fonction de la pulsation de broutement}

On exprime ici la profondeur de passe axiale limite $A_{\text {plim }}$ pour un mode, en fonction de la fréquence de broutement $\omega_{\mathrm{c}}$ (fréquence de vibration de la pièce). Nous utilisons un modèle en coupe orthogonale, bien que la forme des lobes ne soit pas tout à fait la même avec un modèle en coupe oblique. Mais étant donné les imprécisions de mesure sur certains paramètres, nous préférons utiliser un modèle en coupe orthogonale, plus facile à mettre en œuvre, et qui peut être recalé avec des essais. Le déplacement est considéré suivant $y$, et nous étudions le cas du fraisage en avalant.

$$
A_{\mathrm{plim}}=\frac{1}{\left(\frac{z}{2 \pi}\right) \alpha_{y y} K_{\mathrm{t}} \Re e\left[G_{y}\left(i \omega_{\mathrm{c}}\right)\right]}
$$

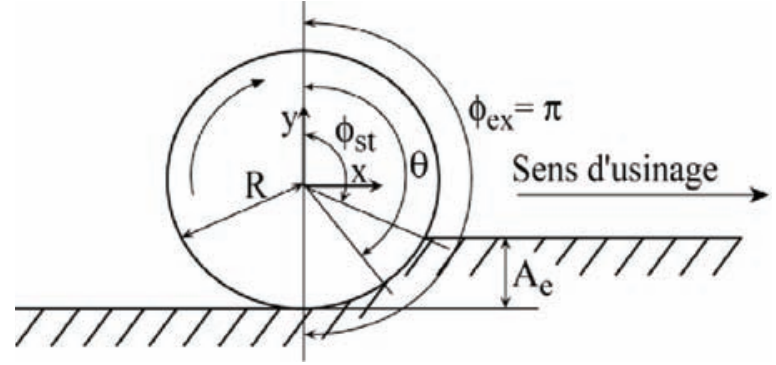

Fig. 3. Angles d'entrée et de sortie en avalant.

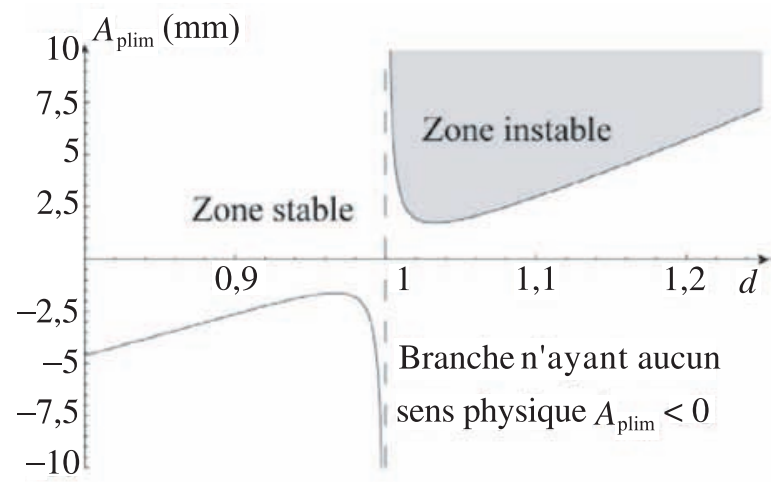

Fig. 4. Tracé de $A_{\text {plim }}$ en fonction de $d$.

Tableau 1. Paramètres utilisés pour tracer le graphe figure 4.

\begin{tabular}{cc}
\hline Pièce en acier (S235) & $A_{\mathrm{e}}=0,1 \mathrm{~mm}$ \\
\hline$R=4 \mathrm{~mm}$ & $z=4$ \\
$K_{\mathrm{t}}=2400 \mathrm{MPa}$ & $K_{\mathrm{r}}=0,9$ \\
$k=45 \times 10^{9} \mathrm{~N} \cdot \mathrm{m}^{-1}$ & $\xi=0,033$ \\
$\omega_{0}=592 \mathrm{~Hz}$ & \\
\hline
\end{tabular}

avec $z$ le nombre de dents de l'outil.

$\alpha_{y y}$ est le coefficient directionnel dynamique suivant $y$ donné par :

$$
\alpha_{y y}=\frac{1}{2}\left[-\cos (2 \theta)-2 K_{\mathrm{r}} \theta-K_{\mathrm{r}} \sin (2 \theta)\right]_{\phi_{\mathrm{st}}}^{\phi_{\mathrm{ex}}}
$$

avec $\theta$ l'angle d'engagement de l'outil, $\phi_{\text {ex }}$ l'angle de sortie de l'outil, et $\phi_{\text {st }}$ l'angle d'entrée de l'outil (Fig. 3).

$\Re e\left[G_{y}\left(i \omega_{c}\right)\right]$ est la partie réelle de la fonction de transfert complexe d'un système à un degré de liberté :

$$
\Re e\left[G_{\mathrm{y}}\left(i \omega_{\mathrm{c}}\right)\right]=\frac{1}{k}\left[\frac{1-d^{2}}{\left(1-d^{2}\right)^{2}+4 \xi^{2} d^{2}}\right]
$$

où $d=\omega_{\mathrm{c}} / \omega_{0}, \omega_{0}$ est la pulsation propre, $k$ est la raideur, et $\xi$ est le taux d'amortissement.

Nous obtenons ainsi le graphe représenté sur la figure 4 .

\subsection{Calcul de la vitesse de rotation de la broche en fonction de la pulsation de broutement}

On cherche ici à exprimer la vitesse de rotation $N$ de la broche en fonction de la pulsation de broutement $\omega_{\mathrm{c}}$. 


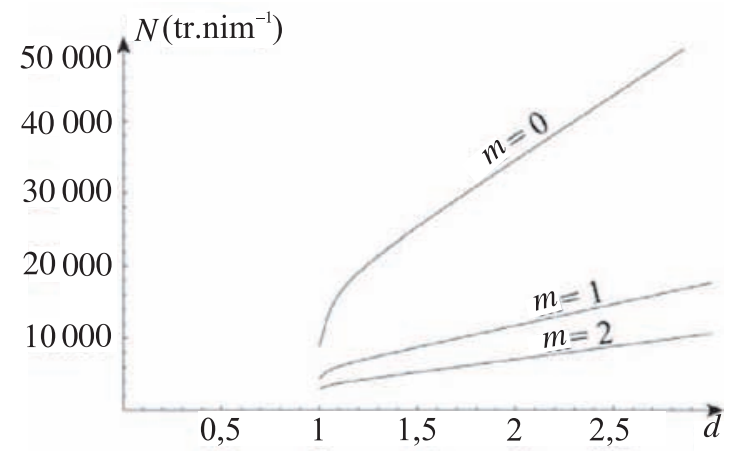

Fig. 5. Tracé de $N$ en fonction de $d$.

À partir de la figure 2, on constate qu'il existe entre deux passages de dent un nombre entier de longueurs d'onde $m$, et une fraction $\varepsilon /(2 \pi)$ de longueur d'onde. En exprimant ce déphasage en fonction de la pulsation de broutement et de la fréquence de dent, nous obtenons l'expression suivante :

$$
N=\frac{60 \omega_{\mathrm{c}}}{z\left[2 m \pi+2 \pi-2 \arctan \left[\frac{d^{2}-1}{2 \xi d}\right]\right]}
$$

L'équation (5) permet de tracer les courbes de la figure 5 , où, pour $m$ fixé, on obtient la vitesse de rotation de la broche correspondant à une pulsation de broutement. Les mêmes paramètres que pour la figure 4 sont utilisés.

\subsection{Calcul de la profondeur de passe axiale limite en fonction de la vitesse de rotation de la broche et du nombre de longueurs d'onde entières}

À ce stade, nous disposons de $A_{\text {plim }}=f\left(\omega_{\mathrm{c}}\right)$ et de $N=f\left(\omega_{\mathrm{c}}\right)$. La fonction $N=f\left(\omega_{\mathrm{c}}\right)$ n'étant pas inversible analytiquement, nous traçons les lobes de manière paramétrique avec $\omega_{\mathrm{c}}$ le paramètre (Fig. 6). Chaque lobe représente la limite de stabilité pour un nombre entier de longueurs d'onde $m$. Les mêmes paramètres que pour la figure 4 et la figure 5 sont utilisés.

Ce calcul est applicable à chaque mode propre de la pièce, lorsque ceux-ci sont suffisamment espacés. La pièce est une structure continue, elle possède donc par définition une infinité de modes propres. Il s'en suit une infinité de lobes. De plus, pour un mode, il peut y avoir une infinité de longueurs d'onde entière entre deux passages de dent. Il y a donc une infinité de lobes par mode. Mais plus le mode considéré est élevé, plus sa raideur est élevée. La profondeur de passe axiale limite étant proportionnel à cette raideur modale ainsi qu'à l'amortissement modal (Éqs. (2), (4)), on s'aperçoit que seuls les premiers modes de la pièce jouent un rôle prépondérant. Sur la figure 7 sont représentés les lobes de stabilité des quatre premiers modes propres de la pièce d'essai. On peut s'apercevoir qu'il n'est pas nécessaire de prendre en compte le quatrième mode, étant donné que la limite fixée par ce mode n'est jamais atteinte.

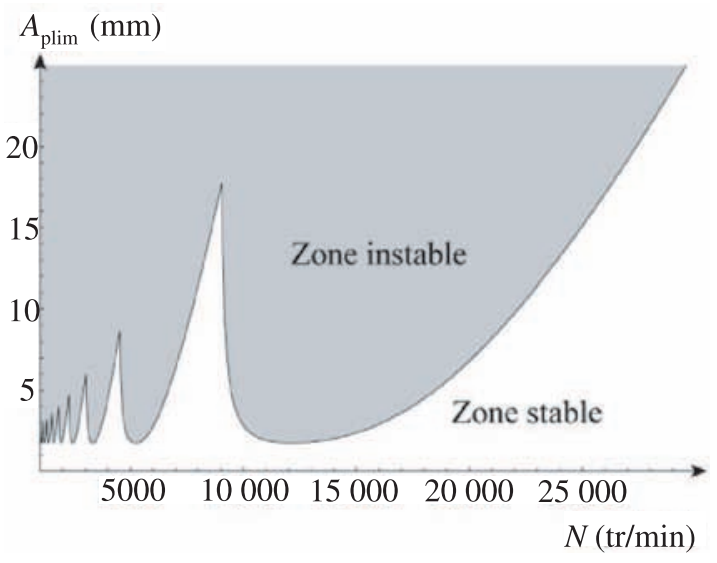

Fig. 6. Tracé caractéristique des lobes de stabilité.

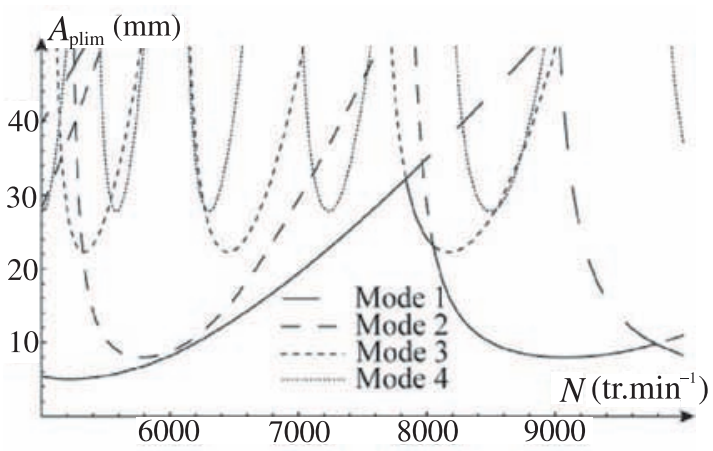

Fig. 7. Tracé des lobes de stabilité pour 4 modes.

Tableau 2. Propriétés dynamiques et conditions de coupe utilisées pour tracer la figure 7 .

\begin{tabular}{clll}
\hline Mode & $\omega_{0}(\mathrm{~Hz})$ & $\xi$ & $k\left(\mathrm{~N}^{-1} \mathrm{~m}^{-1}\right)$ \\
\hline 1 & 592 & 0,033 & $44,497 \times 10^{9}$ \\
2 & 1088 & 0,023 & $108,109 \times 10^{9}$ \\
3 & 2100 & 0,01 & $803,759 \times 10^{9}$ \\
4 & 3320 & 0,009 & $1,119 \times 10^{12}$ \\
Pièce en & $R=4 \mathrm{~mm}$ & $z=4$ & \\
acier (S235) & & & \\
$K_{\mathrm{t}}=2400 \mathrm{MPa}$ & $K_{\mathrm{r}}=0,9$ & $A_{\mathrm{e}}=0,1 \mathrm{~mm}$ & \\
\hline
\end{tabular}

\section{Obtention des paramètres du modèle}

Le calcul et le tracé des lobes de stabilité nécessitent la détermination des paramètres dynamiques de la pièce (fréquences propres, taux d'amortissement, raideurs). Les raideurs et les fréquences propres sont déterminées par un calcul éléments finis. Les taux d'amortissements ne pouvant pas être déterminés facilement par le calcul, nous les déterminons par la mesure.

Nous utilisons un vibromètre laser, avec lequel il n'y a pas contact avec la pièce, pour ne pas perturber la mesure. Ce capteur nous permet de mesurer les fréquences propres sur la machine, et en excitant la pièce avec un marteau d'impact, de déterminer les taux d'amortissements (Fig. 8). Cette mesure nous permet également de 


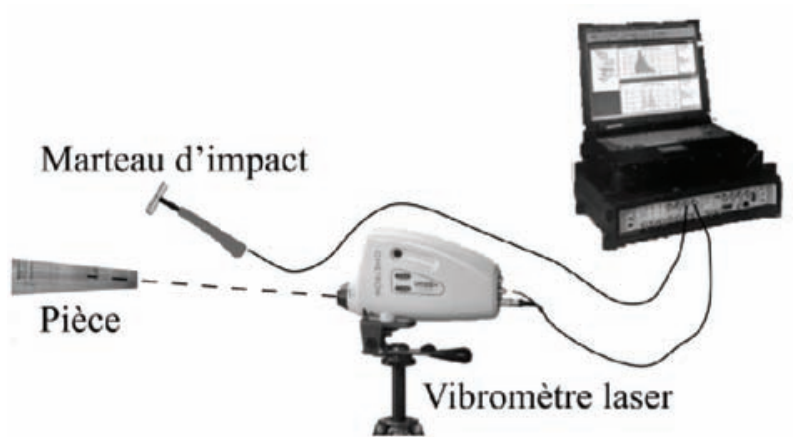

Fig. 8. Dispositif de détermination des paramètres dynamiques.

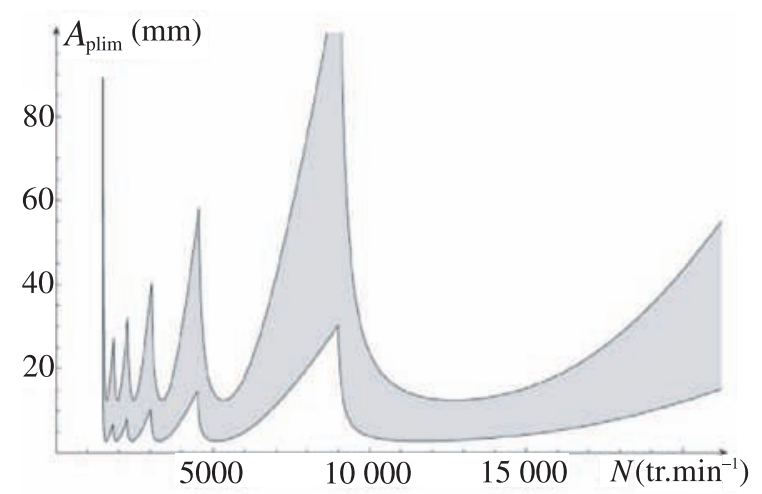

Fig. 9. Tracé des lobes de stabilité intégrant l'imprécision de mesure des paramètres.

Tableau 3. Paramètres utilisés pour tracer le graphe figure 9.

$\omega_{0}=592 \mathrm{~Hz} \pm 1 \mathrm{~Hz}$
$1134 \mathrm{MPa}<K_{\mathrm{t}}<2880 \mathrm{MPa}$
$0,675<K_{\mathrm{r}}<0,9$
$0,0297<\xi<0,0363$
$41,8 \times 10^{9} \mathrm{~N} \cdot \mathrm{m}^{-1}<k<46,2 \times 10^{9} \mathrm{~N} \cdot \mathrm{m}^{-1}$
$0,095 \mathrm{~mm}<A_{\mathrm{e}}<0,105 \mathrm{~mm}$

vérifier l'exactitude du calcul des raideurs en comparant les fréquences propres mesurées et calculées. Nous pourrions également déterminer les raideurs et les formes propres par la mesure, mais dans un contexte industriel, ce sont en général des résultats déjà disponibles en bureau d'étude.

Étant donné l'imprécision des mesures, nous sommes amenés à définir la limite de stabilité en intégrant une certaine marge d'erreur. Il en résulte deux courbes entre lesquelles se trouve la limite de stabilité réelle du système (Fig. 9). Nous ne cherchons pas des précisions plus élevées que celles données dans le tableau 3, notre but étant de faire apparaître des zones d'optimisation.

\section{Influence de la position de l'outil sur le comportement dynamique de la pièce}

Lorsqu'on usine une paroi fine, on se rend compte que la surface résultante n'est pas homogène en terme d'état de surface. Il apparaît des zones fortement marquées et des zones faiblement marquées (Fig. 14). La pièce ne peut donc pas être assimilée à un corps rigide dans la zone usinée, et son comportement dynamique dépend de la position de l'outil. Nous introduisons ainsi une troisième dimension au tracé des lobes de stabilité.

Nous introduisons dans la troisième dimension le point d'application de l'effort de coupe, qui détermine comment chaque mode est excité. Par exemple, le comportement dynamique d'un mode est différent si la force d'excitation est appliquée sur un nœud ou sur un ventre.

Rappelons l'équation dynamique de départ pour le calcul des lobes.

$$
\ddot{U}+2 \xi \omega_{0} \dot{U}+\omega_{0}^{2} U=\left(\left(\underline{F}_{\mathrm{c}} \cdot \underline{y}\right) \omega_{0}^{2}\right) / k
$$

Le déplacement $\underline{u}$ d'un point $\underline{x}$ de la structure, représentée par ces $n$ premiers modes, est défini par :

$$
\underline{u}(\underline{x}, t)=\sum_{i}^{n} \underline{u}_{i}(\underline{x}, t)
$$

avec

$$
\underline{u}_{i}(\underline{x}, t)=\alpha_{i}(t) \underline{\Phi}_{i}(\underline{x})
$$

avec $\underline{\Phi}_{i}(\underline{x})$ la déformée modale du mode $i$, et $\alpha_{i}(t)$ une fonction du temps.

L'équation modale pour chaque mode est :

$$
\ddot{\alpha}_{i}(t)+2 \xi \omega_{i} \dot{\alpha}_{i}(t)+\alpha_{i}(t) \omega_{i}^{2}=\frac{\int_{\partial \Omega} \underline{\Phi}_{i}(\underline{x}) \underline{f}(\underline{x}, t) \mathrm{d} S}{\int_{\Omega} \rho \underline{\Phi}_{i}(\underline{x}) \underline{\Phi}_{i}(\underline{x}) \mathrm{d} \Omega}
$$

avec $\omega_{i}$ la pulsation propre du mode $i, \underline{f}(\underline{x}, t)$ le champ d'effort, et $\int_{\Omega} \rho \underline{\Phi}_{i}(\underline{x}) \underline{\Phi}_{i}(\underline{x}) \mathrm{d} \Omega$ la masse modale $m_{i}$.

En fait, $f(\underline{x}, t)$ est assimilable à un effort ponctuel au point $M(t)$, qui est mobile suivant $\underline{x}_{1}$. C'est l'effort de coupe $F_{\mathrm{c}}(t)$ (Fig. 10).

Ainsi, dans l'équation (9) :

$$
\int_{\Omega} \underline{\Phi}_{i}(\underline{x}) \underline{f}(\underline{x}, t) \mathrm{d} \Omega=\underline{\Phi}_{i}(M(t)) \underline{F}_{\mathrm{c}}(t)
$$

En normalisant les modes en déplacement au point $M_{0}, \underline{\Phi}_{i}\left(M_{0}\right)=1$ et en remplaçant dans les équations (7) et (8) :

$$
\underline{u}_{i}\left(M_{0}, t\right)=\alpha_{i}(t)=\frac{\underline{u}_{i}(M(t), t)}{\underline{\Phi}_{i}(M(t), t)}
$$

Ainsi, par substitution dans l'équation (9), nous obtenons en $M(t)$ :

$$
\begin{aligned}
\underline{\ddot{u}}_{i}(M(t), t)+2 \xi_{i} \omega_{i} \underline{\dot{u}}_{i}(M(t), t)+ & \underline{u}_{i}(M(t), t) \omega_{i}^{2} \\
& =\frac{\underline{\Phi}_{i}^{2}(M(t)) \underline{F}_{c}(t)}{m_{i}}
\end{aligned}
$$




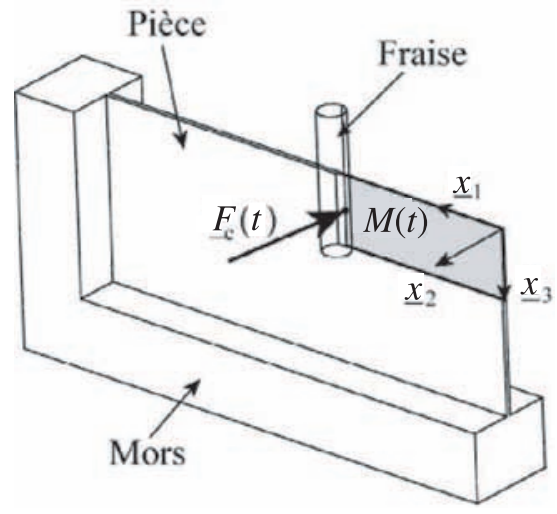

Fig. 10. Application de l'effort de coupe sur la pièce d'essai.

L'équation (12) est analogue à l'équation (6) de départ, avec :

$$
\begin{gathered}
U=\underline{u}_{i}(M(t), t) \cdot \underline{x}_{2} \\
\xi=\xi_{i} \\
\omega_{0}=\omega_{i} \\
k=\frac{k_{i}}{\underline{\Phi}_{i}^{2}(M(t))}
\end{gathered}
$$

$k_{i}, \omega_{i}$ et $\underline{\Phi}_{i}(M(t))$ sont obtenus par un calcul éléments finis. Ils peuvent être également déterminés par la mesure.

Le modèle de coupe avec vibrations régénératives est valable en régime établi. Ainsi, le temps nécessaire pour observer une variation significative de $k_{i}$ doit être beaucoup plus long que le temps nécessaire au système pour atteindre le régime établi. Les essais d'usinage montrent que la transition de stabilité est très rapide par rapport à la variation de la raideur $k$ (Éq. (16)) (Fig. 14).

Nous pouvons donc tracer les lobes de stabilité pour tous les modes propres de la pièce en tout point de la trajectoire de l'outil. Pour un mode, la limite de stabilité étant proportionnelle à la raideur $k$ (Éqs. (2), (4)), la limite de stabilité augmente lorsque l'outil est sur un nœud. La raideur $k$ est théoriquement infinie sur un nœud (Éq. (16)) (Fig. 12).

\section{Essais de validation}

L'étude porte sur l'usinage en contournage et en avalant d'une plaque en acier d'épaisseur $1 \mathrm{~mm}$, encastrée sur deux de ses côtés perpendiculaires (Fig. 10). La profondeur de passe radiale programmée est de $0,1 \mathrm{~mm}$ et l'avance à la dent de 0,05 mm.dent ${ }^{-1}$. Nous utilisons une fraise cylindrique de $8 \mathrm{~mm}$ de diamètre, à 4 dents et avec un angle d'hélice de $45^{\circ}$.

Les lobes de stabilité correspondant à cette pièce sont représentés sur la figure 11 pour les modes 1 et 2 , avec les mêmes paramètres que pour la figure 4 . Ils sont tracés au point $M_{0}$ de la pièce qui correspond au coin libre.

Nous réalisons un usinage à profondeur de passe axiale (et radiale) constante en choisissant une vitesse

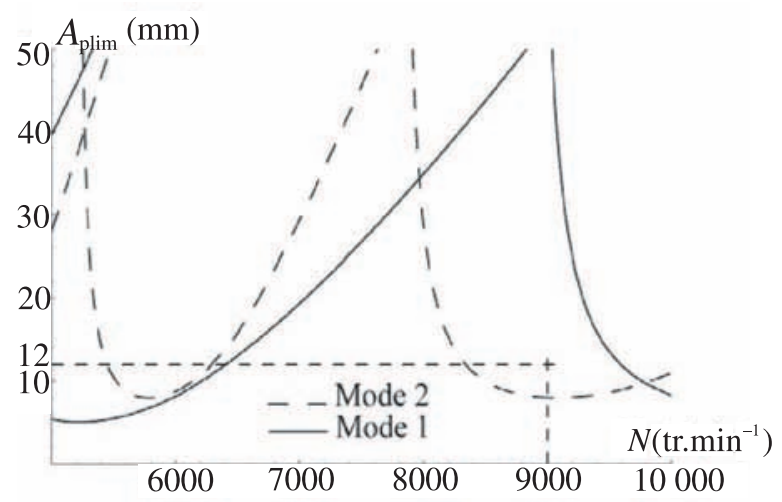

Fig. 11. Tracé des lobes des 2 premiers modes de la pièce d'essai.

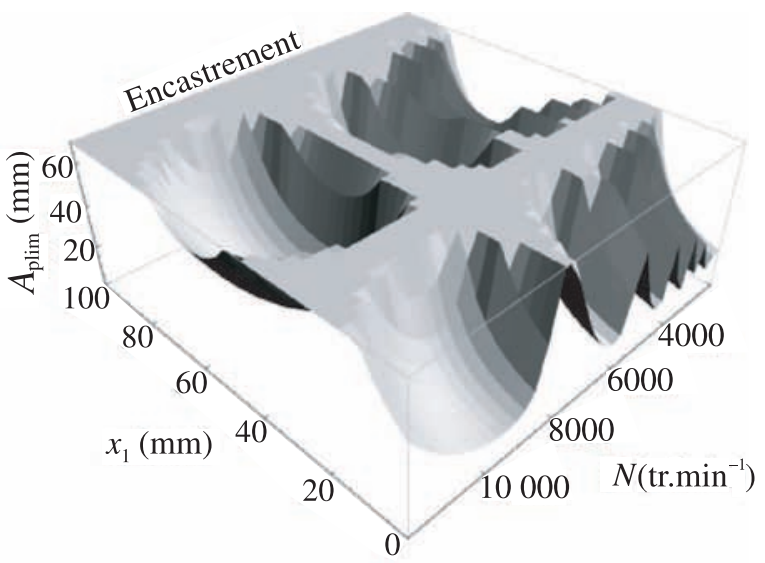

Fig. 12. Tracé des lobes en trois dimensions du $2^{\mathrm{e}}$ mode de la pièce.

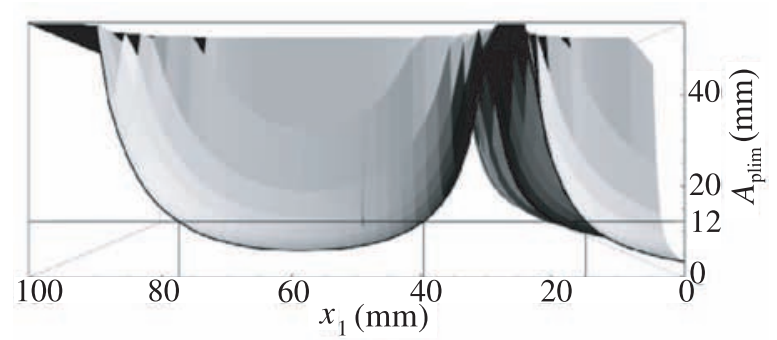

Fig. 13. Coupe à $N=9000$ tr.min ${ }^{-1}$ dans le plan $\left(x_{1}, A_{\text {plim }}\right)$.

de rotation volontairement instable au point $M_{0}$ en cherchant à exciter le deuxième mode de la pièce, soit $N=9000$ tr.min ${ }^{-1}$, et $A_{\mathrm{p}}=12 \mathrm{~mm}$. Le tracé des lobes de stabilité en 3 dimensions du deuxième mode de la pièce est représenté sur la figure 12.

Une coupe du tracé des lobes en 3 dimensions dans le plan $\left(x_{1}, A_{\text {plim }}\right)$ à la vitesse de 9000 tr.min ${ }^{-1}$, nous permet de déterminer les zones d'apparition des vibrations sur la pièce (Fig. 13). Nous réalisons également un usinage en augmentant progressivement la profondeur de passe axiale de façon à faire apparaître la limite de stabilité en un point de la pièce et ainsi recaler le tracé selon l'axe $A_{\text {plim }}$. 


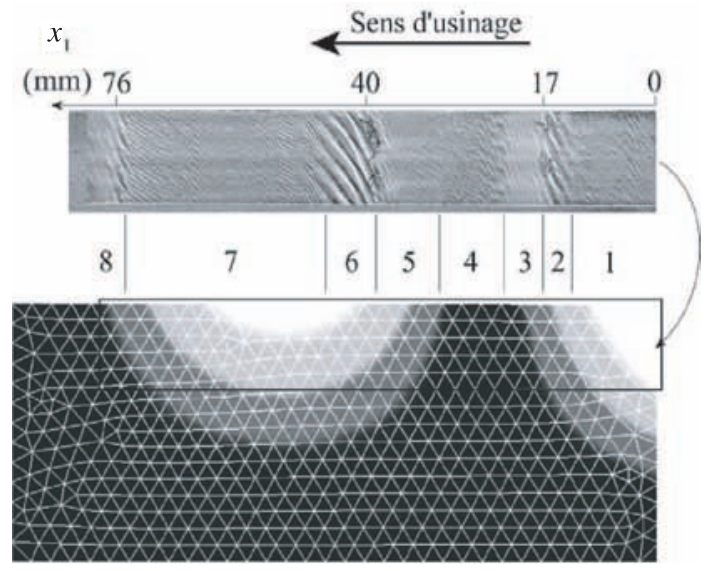

Fig. 14. Position des transitions stabilité/instabilité sur la surface usinée et corrélation avec la déformée propre du deuxième mode.

On constate une bonne corrélation entre la position des transitions stabilité/instabilité prédite par les lobes et celle observée sur la surface résultant de l'usinage (Fig. 14).

De plus, en confrontant cette surface à la déformée modale du deuxième mode obtenue par simulation (Fig. 14), on retrouve bien les nœuds (zone 4) et les ventres (zones 1 et 7) du mode considéré. Par contre on observe différentes zones de transition sur cette surface.

La zone 4 se trouve sur le noud du mode, et on constate que l'état de surface est relativement acceptable. L'usinage dans cette zone est stable, comme l'ont prédit les lobes en 3 dimensions. Les zones 1 et 7 se trouvent sur les ventres du mode, la pièce présente un comportement très instable et fortement non-linéaire lors de l'usinage, même si l'état de surface paraît acceptable. Il est dès lors très difficile de prédire le comportement de la pièce, l'outil sortant de la matière $[11,12]$. Le passage d'une zone instable à une zone stable (et inversement) se fait en fait en 2 étapes distinctes. La première étape (zones 2, 6 et 8) présente un état de surface très dégradé dont les marques traduisent une atténuation (ou augmentation) des vibrations en terme d'amplitude et de fréquence par rapport aux zones instables. Les marques observées sont très ordonnées, ce qui est dû au fait que l'outil sort de la matière d'une part, et que la fréquence de vibration de la pièce tend à s'accorder avec un multiple de la fréquence de dent d'autre part. La deuxième étape (zones 3 et 5) montre un comportement plus calme de la pièce. La fréquence de vibration est stabilisée, et l'outil ne sort plus de la matière. On observe seulement une atténuation (ou augmentation) de l'amplitude.

Nous ne pousserons pas plus loin l'étude des différentes marques observées sur la surface résultant de l'usinage, car les renseignements qu'elles nous fournissent (fréquence et amplitude de vibration) peuvent être obtenu par d'autres moyens dont nous parlerons dans le prochain chapitre. Comme nous l'avons vu plus haut, l'état de surface observé dans les zones instables peut être du même ordre que celui observé dans les zones stables, voire meilleur. Mais dans les zones instables il est très difficile de maîtriser la profondeur de passe radiale, donc les contraintes dimensionnelles. De plus, l'apparition de zones instables passe nécessairement par des zones de transition très perturbées autour des nœuds de la pièce. Ces zones de transition ne respectant pas les contraintes d'état de surface, nous ne chercherons donc pas à tirer parti des zones instables.

\section{Mesure des vibrations pendant l'usinage}

Pour mesurer les vibrations pendant l'usinage, il existe différentes méthodes dont la mesure de l'intensité acoustique proposée par T. Delio et al. [13]. Mais cette méthode nécessite des filtres d'atténuation des bruits incompatibles avec l'environnement d'un atelier de fabrication. Elle s'avère donc difficile à mettre en œuvre dans ce contexte. C'est pourquoi, la chaîne de mesure décrite plus haut, nécessaire à l'obtention des paramètres dynamiques de la pièce, va également nous permettre de mesurer les vibrations de la pièce pendant l'usinage. Par contre, cette mesure est incompatible avec l'utilisation de la lubrification. En effet, le faisceau est perturbé par le fluide de coupe. Il s'agit donc d'effectuer des usinages à sec pour pouvoir mesurer les vibrations. Mais nous savons par expérience que le fluide de coupe augmente légèrement l'amortissement du système usinant. Dès lors, un usinage stable à sec, le sera d'autant plus avec la lubrification.

Pour mesurer les vibrations, nous enregistrons le signal dans le domaine temporel pendant toute la durée de l'usinage grâce à un logiciel de traitement du signal. Le logiciel nous permet ensuite de sélectionner la partie du signal correspondant à la zone désirée, et d'effectuer une FFT afin d'obtenir les différentes fréquences de vibration de la pièce dans cette zone. Nous pouvons ainsi caractériser chaque zone en terme de fréquence et d'amplitude.

\section{Perspectives}

Par définition, l'usinage est un procédé de mise en forme par enlèvement de matière. Ce qui induit, lorsque l'enlèvement de matière est significatif, une modification des paramètres dynamiques de la pièce (fréquences propres notamment) fonction de la position de l'outil. Ainsi, on intègre, sur la troisième dimension introduite plus haut, en plus du changement de comportement au passage de l'outil sur les nœuds et les ventres, la variation des paramètres dynamiques de la pièce. La modification des fréquences propres de la pièce pendant l'usinage introduit un décalage des lobes suivant l'axe portant la vitesse de rotation de la broche. Dès lors, si ce décalage est assez important, il peut arriver qu'il n'existe pas de vitesse de rotation stable pour tout l'usinage. Il faut alors pouvoir faire varier cette vitesse en cours d'usinage de façon à assurer la complète stabilité du système.

Au début de cet article, nous avons considéré que l'outil est infiniment rigide au regard de la pièce. Mais il arrive 
que cette hypothèse ne soit pas vérifiée, et que la pièce et l'outil soient mobiles. Auquel cas, si les modes propres de la pièce et de l'outil sont proches, il peut y avoir couplage entre les deux. La théorie des lobes de stabilité telle que nous l'avons présentée n'est plus valable, et il faut réécrire les équations suivant la nouvelle configuration.

Nous avons également considéré l'outil comme étant cylindrique où la vitesse de coupe est constante en tout point de la périphérie de celui-ci, et où l'angle d'hélice est constant. La répartition de l'effort de coupe est donc uniforme le long de l'outil, et nous connaissons la position de la résultante de cet effort réparti. Mais nous allons être amené à utiliser un outil conique à angle d'hélice variable. La répartition de l'effort n'est plus uniforme dans ce cas là, et la position de la résultante de l'effort de coupe change.

Nous allons également être confronté au cas où nous usinons une pièce mince avec un outil conique déformable (usinage d'un rouet centrifuge par exemple) avec un enlèvement de matière significatif.

\section{Conclusion}

Dans cet article, nous avons montré comment introduire dans la théorie des lobes de stabilité les variations de comportement dynamique de la pièce, en fonction de la position de l'outil. Une troisième dimension est ainsi intégrée au tracé des lobes, qui est la position de l'outil. Cette représentation des lobes en trois dimensions est validée par des usinages expérimentaux. Nous pouvons ainsi obtenir des conditions de coupe optimales tout le long de l'usinage.

Il est également nécessaire d'intégrer à la théorie des lobes de stabilité la variation des paramètres dynamiques de la pièce pendant l'usinage dû à l'enlèvement de matière, ainsi que l'utilisation d'outils déformables. Ce travail est en cours et sera développé plus tard. Le modèle ainsi obtenu devra faire l'objet soit d'une intégration à la CFAO, soit à la mise au point d'un logiciel dédié adapté au contexte industriel.

\section{Références}

[1] S.A. Tobias, W. Fishwick, A Theory of Regenerative Chatter, The Engineer (1958)

[2] J. Tlusty, M. Polacek, The stability of Machine Tools Against Self Excited Vibrations in Machining, Int. Res. Production Eng., ASME (1963) 465-474

[3] H.E. Merritt, Theory of Self-Excited Machine Tool Chatter, ASME J. Eng. Industry 87 (1965) 447-454

[4] R. Sridhar, R.E. Hohn, G.W. Long, General Formulation of the Milling Process Equation, ASME J. Eng. Industry (1968a) 317-324

[5] R. Sridhar, R.E. Hohn, G.W. Long, A Stability Algorithm for the General Milling Process, ASME J. Eng. Industry (1968b) 330-334

[6] Y. Altintas, E. Budak, Analytical Prediction of Stability Lobes in Milling, Annals of the CIRP 44/1 (1995) 357-362

[7] F. Lapujoulade, T. Mabrouki, K. Raïssi, Prédiction du Comportement Vibratoire du Fraisage Latéral de Finition des Pièces à Parois Minces, Mécanique \& Industries 3 (2002) 403-418

[8] M.A. Davies, B. Balachandran, Impact Dynamics in Milling of Thin Walled Structures, Nonlinear Dynamics 22 (2000) 375-392

[9] E. Budak, Y. Altintas, Analytical Prediction of Chatter Stability in Milling, Part I : General Formulation, Transaction of the ASME 120 (1998) 22-30

[10] E. Budak, Y. Altintas, Analytical Prediction of Chatter Stability in Milling, Part II : Application of the General Formulation to Common Milling Systems, Transaction of the ASME 120 (1998) 31-36

[11] M.S. Fofana, Effect of Regenerative Process on the Sample Stability of a Multiple Delay Differential Equation, Chaos, Solitons \& Fractals 14 (2002) 301-309

[12] I. Minis, T. Yanushevsky, R. Tembo, R. Hocken, Analysis of Linear and Nonlinear Chatter in Milling, Annals of the CIRP 39 (1990) 459-462

[13] T. Delio, S. Smith, J. Tlusty, Use of Audio Signals Chatter Detection and Control, J. Eng. Industry 114 (1992) 27-34 